EPJ Web of Conferences 41, 04035 (2013)

DOI: $10.1051 /$ epjconf/20134104035

(C) Owned by the authors, published by EDP Sciences, 2013

\title{
Absolute femtosecond measurements of Auger recombination dynamics in lead sulfide quantum dots
}

\author{
B. Cho ${ }^{1}$, W. K. Peters ${ }^{1}$, V. Tiwari ${ }^{1}$, A. P. Spencer ${ }^{1}$, D. Baranov ${ }^{1}$, R. J. Hill ${ }^{1}$ and D. M. Jonas ${ }^{1}$ \\ ${ }^{1}$ Department of Chemistry and Biochemistry, University of Colorado, Boulder, Colorado 80309- \\ 0215, USA
}

\begin{abstract}
Multiphoton excitation of hot carriers generates multiexcitons that are probed as a function of the absolute number of photons absorbed. Standard assumptions of Auger recombination analyses fail for an average of 2 excitations.
\end{abstract}

\section{Introduction}

Quantum dots have attracted considerable attention because of the potentially useful properties stemming from quantum confinement [1]. For example, multiple exciton generation (MEG) has been reported in PbS dots [2]. However, there is still an on-going controversy over the mechanism and yield of MEG [3]. The reverse process to MEG is the less controversial Auger recombination (AR); a standard model based on stepwise annihilation of multiexcitons exists [4]. This Auger analysis assumes that the signal is proportional to the number of electron-hole pairs, $\mathrm{N}_{\mathrm{eh}}$, that photo-excitation follows Poisson statistics, and that there are no annihilation processes other than AR. Poisson statistics can predict the initial and final signals with the microscopic kinetic model for AR connecting the two ends of the signals. $\mathrm{PbS}$ dots have bandgap degeneracy of 8; at high excitation intensities, "band-filling" was invoked to explain a rapid Auger process not described by the standard model [5]. However, $<\mathrm{N}_{\mathrm{eh}}>$ was not known absolutely. For $8 \mathrm{~nm} \mathrm{PbS}$ dots, we have measured pump-probe transients up to an absolutely known $<\mathrm{N}_{\mathrm{eh}}>=10( \pm 10 \%)$, where the brackets around $\mathrm{N}_{\mathrm{eh}}$ indicate a weighted average over the probe beam profile. The assumptions of the standard Auger analysis fail below $<\mathrm{N}_{\mathrm{eh}}>=2$.

\section{Experimental}

The pump-probe experiments in Figure 1 used pulses from a home-built noncollinear optical parametric amplifier delivering $150 \mathrm{~nJ}, 35 \mathrm{fs}, 500 \mathrm{~nm}$ pulses at $10 \mathrm{kHz}$ repetition rate. The NOPA was pumped by a Coherent RegA seeded by a home-built Ti:Sapphire oscillator running at $76 \mathrm{MHz}$. AR experiments used a Coherent RegA delivering $750 \mathrm{~nJ}, 75 \mathrm{fs}, 800 \mathrm{~nm}$ pulses at $20 \mathrm{kHz}$ repetition rate. The oleate capped $\mathrm{PbS}$ dots were supplied by NREL and were dispersed in toluene before use. The dot samples were held in an air-tight spinning cell (1800 rpm) to ensure that the sample is refreshed after each laser shot. The pump-probe signal was measured by a Si photodiode using lock-in detection. For fluorescein, calculation of the absolute signal size utilized the steady state absorption and emission spectrum, the transverse

This is an Open Access article distributed under the terms of the Creative Commons Attribution License 2.0, which permits unrestricted use, distribution, and reproduction in any medium, provided the original work is properly cited. 
spatial profile of the laser beams, the pulse spectra, and the known electronic state lifetime. Figure 1 shows the calculated and measured time-dependent pump-probe signal for fluorescein at magic angle pump-probe polarization. Agreement to within 5\% indicates the accuracy for $\mathrm{N}_{\mathrm{eh}}$.
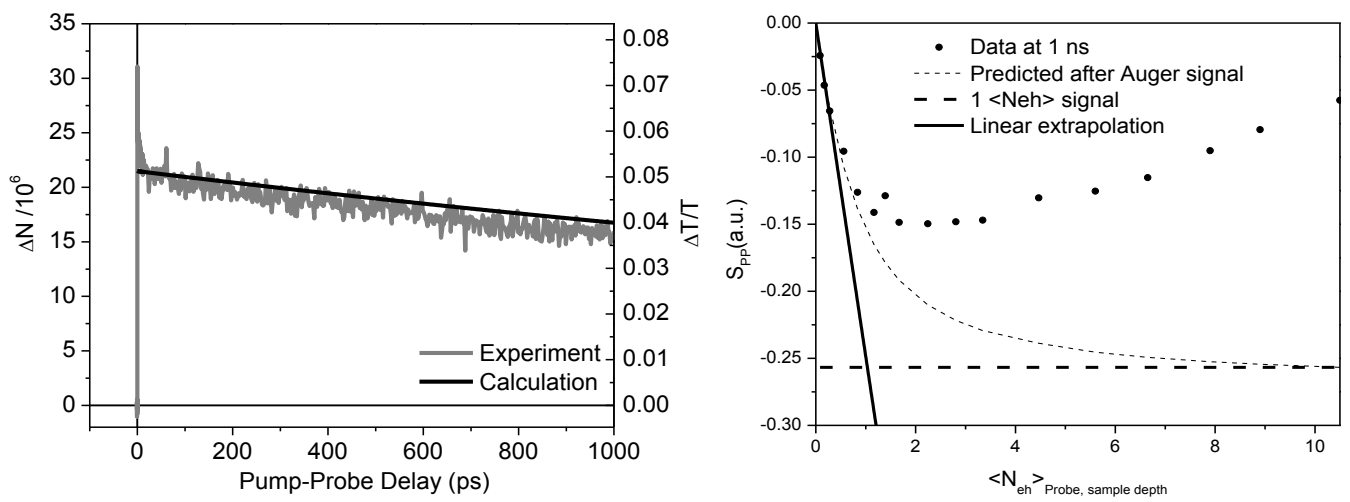

Fig.1. (left) Absolute change in the number of probe photons transmitted through fluorescein dianion in basic methanol for a magic angle pump-probe transient as a function of pump-probe delay. The experimental measurement (grey) is compared to a calculation without any adjustable parameters (black), verifying the absolute number of pump photons absorbed.

Fig. 2 (right) Pump-probe signal for $8 \mathrm{~nm} \mathrm{PbS}$ dots averaged from $700 \mathrm{ps}$ to $1 \mathrm{~ns}$ (solid circle) as a function of $\left\langle\mathrm{N}_{\mathrm{eh}}>\right.$. The solid black line is the linear extrapolation from the data at low $<\mathrm{N}_{\mathrm{eh}}>$. The short dashed curve assumes all excited dots, regardless of $\mathrm{N}_{\mathrm{eh}}$, end up with $\mathrm{N}_{\mathrm{eh}}=1$ (complete AR). At high $\left\langle\mathrm{N}_{\mathrm{eh}}\right\rangle$, the short dashed curve approaches the linear extrapolation of the low $<\mathrm{N}_{\mathrm{eh}}>$ data to $\left\langle\mathrm{N}_{\mathrm{eh}}>=1\right.$ (long dashed horizontal line): Data (dots) show the assumptions of the standard Auger analysis fail below $<\mathrm{N}_{\mathrm{eh}}>=2$.

\section{Results and Discussion}

Ordinarily, the $\mathrm{PbS}$ dot emission lifetime is $\sim 300$ ns and biexciton Auger recombination lifetime is $\sim 200$ ps. Figure 2 shows pump-probe signals at 1 ns delay (after AR is complete but before population loss is expected) as a function of $\left\langle\mathrm{N}_{\mathrm{eh}}>\right.$. Because $\left\langle\mathrm{N}_{\mathrm{eh}}\right\rangle$ is absolutely known, the height of the long dashed line is known a priori, and a discrepancy with the standard Auger analysis assumptions is revealed by $\left\langle\mathrm{N}_{\mathrm{eh}}\right\rangle=1.5$. Figure 3 shows that the pump-probe signal at 100 fs delay is linear in $\left\langle\mathrm{N}_{\mathrm{eh}}\right\rangle$ up to $\left\langle\mathrm{N}_{\mathrm{eh}}\right\rangle=10$. This likely indicates that photo-excitation generates a Poisson distribution and that the signal is proportional to $\mathrm{N}_{\mathrm{eh}}$ before relaxation. This is an advantage of exciting hot carriers that interact only weakly; these assumptions of the Auger analysis do not hold for band edge excitation of excitons. A likely explanation for the discrepancies in Figure 2 is charge ejection from the dot into a surface or ligand trap[6, 7]. Charge separated dots have an emission lifetime of $\sim 300 \mathrm{ps}$; the signal at $1 \mathrm{~ns}$ can be explained by assuming charge separation in $\sim 4 / 5$ of the dots at $<\mathrm{N}_{\mathrm{eh}}>=10$.

Figure 3 also shows that the pump-probe signal at 1.5 ps (after carrier cooling) starts to saturate at $\left\langle\mathrm{N}_{\mathrm{eh}}\right\rangle \approx 4$. The early time development of this saturation is shown as a function of initial $<\mathrm{N}_{\mathrm{eh}}>$ and pump-probe delay on Figure 4. This is possible because the signal is linear in $<\mathrm{N}_{\mathrm{eh}}>$ up to $\sim 200 \mathrm{fs}$ delay. At present it is not yet clear whether these fast saturation dynamics are connected to charge separated states (which could arise from persistent charge separated states with lifetimes greatly exceeding the $30 \mathrm{~ms}$ time required for the sample to rotate back into the beam) or band-filling. 

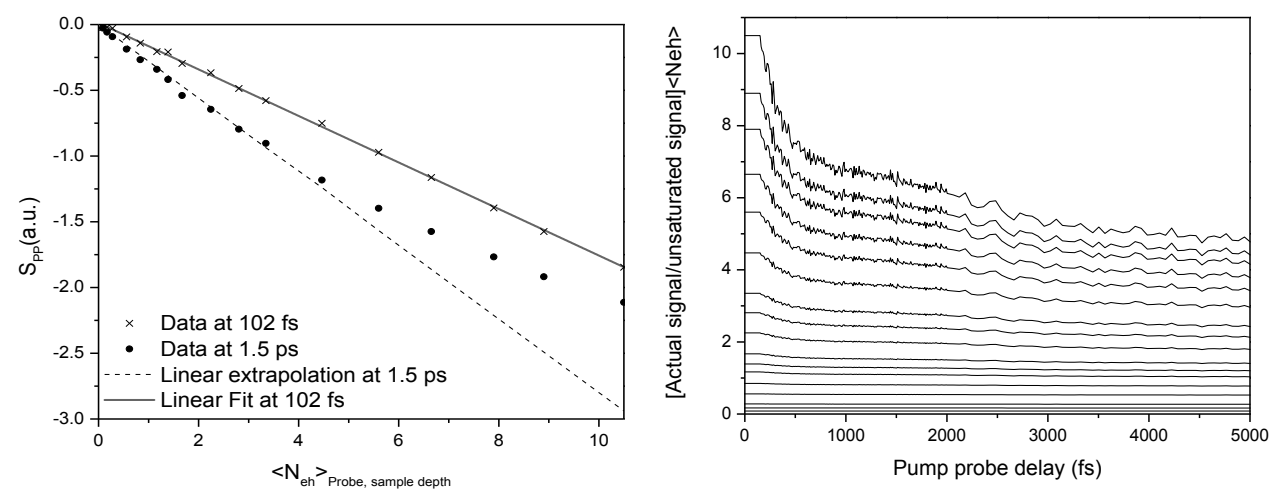

Fig.3 (left) $8 \mathrm{~nm}$ diameter PbS dot pump-probe signals at $102 \mathrm{fs}$ (cross) and $1.5 \mathrm{ps}$ (solid circle) as a function of $<\mathrm{N}_{\mathrm{eh}}>$.

Fig.4 (right) Saturation of the pump-probe signal (signal divided by the linear extrapolation from the low power signal at each pump-probe delay) as a function of pump-probe delay. Since the signal for delays near time zero is proportional to $\left\langle\mathrm{N}_{\mathrm{eh}}\right\rangle$, each curve was multiplied by the initial $<\mathrm{N}_{\mathrm{eh}}>$.

\section{Conclusion}

Multiexciton dynamics with an absolute $\mathrm{N}_{\text {eh }}$ scale shows that the assumptions of the standard Auger analysis have limited validity beyond $\left\langle\mathrm{N}_{\mathrm{eh}}\right\rangle=1$. The dot quenching processes revealed by scanning to $1 \mathrm{~ns}$ delay and absolutely calibrating $\left\langle\mathrm{N}_{\mathrm{eh}}>\right.$ may affect the early time dynamics previously attributed to band filling. Close inspection of Fig. 3 indicates time-dependent saturation within 1 ps at around $<\mathrm{N}_{\mathrm{eh}}>=0.5$; this is faster than ordinary AR or trion decay.

\section{Acknowledgment}

This work was funded by the Division of Chemical Sciences, Geosciences, and Biosciences, Office of Basic Energy Sciences of the U.S. Department of Energy through Grant DE-FG02-07ER15912.

\section{References}

1. F. W. Wise, Acc. Chem. Res. 33, 773 (2000)

2. A. J. Nozik, M. C. Beard, J. M. Luther, M. Law, R. J. Ellingson, and J. C. Johnson, Chem. Rev. 110, $6873(2010)$

3. P. Tyagi and P. Kambhampati, J. Chem. Phys. 134, 094706 (2011)

4. V.I. Klimov, J.A. McGuire, R.D. Schaller, and V.I. Rupasov, Phys. Rev. B 77, 195324 (2008).

5. E. Istrate, S. Hoogland, V. Sukhovatkin, L. Levina, P.W.E. Smith, and E.H. Sargent, J. Phys. Chem. B 112, 2757 (2008).

6. A. G. Midgett, H. W. Hillhouse, B. K. Huges, A. J. Nozik and M. C. Beard, J. Phys. Chem. C 114, $17486(2010)$

7. L. A. Padilha, I. Robel, D. C. Lee, P. Nagpal, J. M. Pietryga and V. I. Klimov, ACS Nano 5, $5045(2011)$ 